\title{
Magnetization-Prepared Rapid Gradient Echo MRI
}

National Cancer Institute

\section{Source}

National Cancer Institute. Magnetization-Prepared Rapid Gradient Echo MRI. NCI

Thesaurus. Code C118462.

A magnetic resonance imaging modality that offers rapid imaging time, easy

reconstruction of any plane, and three-dimensional surface contour rendering with cut away postprocessing. Especially useful for imaging brain, MP-RAGE captures high tissue contrast and provides high spatial resolution with whole brain coverage in a short scan time. 